\title{
ON HALF EXPONENTIAL POWER MODEL FOR THE FIRST TIME FAILURE OF POWER DISTRIBUTION TRANSFORMERS IN NIGERIA*
}

\author{
A. A. Olosunde ${ }^{1 \ddagger}$ and R. B. Ekpo ${ }^{2}$
}

\footnotetext{
${ }^{1}$ Department of Mathematics, Obafemi Awolowo University, Ile-Ife, Nigeria, akinolosunde@gmail.com

${ }^{2}$ Department of Mathematics, Obafemi Awolowo University, Ile-Ife, Nigeria, ekporowland@gmail.com ${ }^{\ddagger}$ corresponding author
}

\section{Indonesian Journal of Statistics and Applications Vol 4 No 2 (2020), 321 - 326}

Copyright (c) 2020 A. A. Olosunde and R. B. Ekpo. This is an open-access article distributed under the Creative Commons Attribution License, which permits unrestricted use, distribution, and reproduction in any medium, provided the original work is properly cited.

\begin{abstract}
Transformer failure is a major problem confronting the Nigerian power sector, hindering the transmission and distribution of electric power to various households, institutions, and industries. Many of these transformer developed problem due to the old age of the transformers, overloading, in-availability of technical expertise, poor maintenance culture, manufacturer's faults, just to mention few. The present research focuses on providing half exponential power model for the failure of already installed transformers, with respect to years of installation up to the time of the first failure, using secondary data from the south western part of Nigeria as a case study. The results obtained showed that half exponential power performed better in modeling the first time failure of power transformers. This was possible because of the present of shape parameter which gives flexibility to half exponential power when compared with a half normal distribution.
\end{abstract}

Keywords: electric power, exponential power distribution, shape parameter, short tails.

*Received Mar 2020; Accepted Jul 2020; Published online on Jul 2020 


\section{Introduction}

In reliability theory, Lawless (2011), Kalbfleisch and Prentice (2011) observed that the common theoretical results about systems failure permits researchers to predict the agerelated failure kinetics for a system of given reliability structure and of its components. This general theory also predicts that when those systems that are totally composed of non-ageing elements (with a constant failure rate) will however fail more often as time goes on, if these systems are redundant in irreplaceable elements. Ageing, therefore, is a direct consequence of systems redundancy.

Reliability theory, in general is important in the application of probability theory to the modelling of failures and the prediction of success probability. We have found the concept useful in the present study because modern probability concepts base many of its results on the concept of a random variable, its pdf's, and the cdf's. In the case of reliability, the random variable of interest is the time to failure, $T$. We develop the basic relationships needed by focusing on the probability that the time to failure $T$ is in the interval $(t, t+\delta t)$.

Definition 1.1 Suppose $X>0$ is a continuous random variable with cumulative density function (cdf), survival function (sf) and probability density function (pdf) as $F(x ; \theta), \bar{F}(x ; \theta)$, and $f(x ; \theta)$ respectively. Where $\theta$ is a vector parameter. The hazard function and reversed hazard function of $X$ will be denoted by

$$
h(x ; \theta)=\frac{f(x ; \theta)}{\bar{F}(x ; \theta)}=-\frac{d}{d x} \ln \bar{F}(x ; \theta) \quad \text { and } \quad r(x ; \theta)=\frac{f(x ; \theta)}{F(x ; \theta)}=-\frac{d}{d x} \ln F(x ; \theta)
$$

respectively.

\section{The Exponential Power Distribution}

The Exponential Power Distribution (EPD) is a generalization of the distribution of random errors. In statistical inference, the usual hypothesis on random errors is that they are distributed as normal distribution, but often this hypothesis is not suitable to the study of natural phenomena. The distribution under present consideration is the exponential power distribution, which has the advantages of thick- and thin- tailed distributions. It is a natural generalization of normal, Laplace and Kotz-symmetric distribution. The mean is the location parameter, therefore, given way for the construction of linear and non-linear models. Several properties and applications of this distribution have been studied by authors see Olosunde (2013) and Olosunde and Adegoke (2016).

The univariate exponential power distribution is given

$$
f(x ; \mu, \sigma, \beta)=\frac{1}{\sigma \Gamma\left(1+\frac{1}{2 \beta}\right) 2^{1+\frac{1}{2 \beta}}} \exp \left\{-\frac{1}{2}\left|\frac{x-\mu}{\sigma}\right|^{2 \beta}\right\}
$$

where the parameters $\mu \in \Re$ and $\sigma \in(0, \infty)$ are respectively scale and location parameters and $\beta \in(0, \beta)$ is the shape parameter which regulates the tails of the distribution such that when $\beta=1$ the density (1) is normal, but for $\beta=1 / 2$ we have 
double exponential distribution. The distribution has been used in robust inference and software have been developed for the estimation of its parameters see Mineo and Ruggieri (2005), The parameters of the distribution were estimated via maximum likelihood estimation method see Agro (1995).

\subsection{The Half-Exponential Power Distribution}

Definition 2.1 Suppose a random variable $x$ has pdf (2). Then we define

$$
g(x)=f(x)+f(-x) \Longrightarrow f(x)+f(x)
$$

$f(x)$ is an even function

$$
g(x)=2 f(x)=\frac{p^{1-\frac{1}{p}}}{\sigma \Gamma\left(\frac{1}{p}\right)} e^{-\frac{x^{p}}{p \sigma^{p}}}, \quad x \geq 0
$$

$x \in(-\infty, 0][0, \infty)$ where $\sigma>0$ and $p>0$ to be half exponential power distribution with scale parameter $\sigma>0$, which regulates the tail of the distribution and makes it more robust in modelling when compare to half normal and exponential distributions commonly used in the literature.

The distribution (3) is denoted as $X \sim \operatorname{HEP}(\sigma, p)$ Where $p>0$ is the shape parameter. Half-exponential distribution was first studied by Gui (2013), several properties of this distribution were presented which makes the distribution appealing for model our life time distribution data of power transformer. The cumulative and the Hazard Function for HEP as given in Gui (2013) are as given below;

The cumulative distribution function of the half exponential power distribution is given as;

$$
F(x)=\int_{0}^{x} f_{x}(u) d u=\int_{0}^{x} \frac{p^{1-\frac{1}{p}}}{\sigma \Gamma \frac{1}{p}} e^{-\frac{u^{p}}{p \sigma^{p}}} d u=\frac{\gamma\left(\frac{1}{p}, \frac{x^{p}}{p \sigma^{p}}\right)}{\Gamma\left(\frac{1}{p}\right)}
$$

where $\gamma($,$) is the lower incomplete gamma function, defined as \gamma(s, x)=\int_{0}^{x} t^{s-1} e^{-t} d t$ The hazard rate function (also known failure rate function) of the half exponential power distribution is given by, for $x \geq 0$

$$
h(t)=\frac{f(x)}{1-F(x)}=\frac{p^{1-\frac{1}{p}} e^{\frac{x^{p}}{p \sigma^{p}}}}{\sigma\left[\Gamma\left(\frac{1}{p}\right)-\gamma\left(\frac{1}{p}, \frac{x^{p}}{p \sigma^{p}}\right)\right]}
$$

In general, there are no explicit solutions for the maximum likelihood estimating equations for the distribution, to estimates the parameter of our data we use a code written in $\mathrm{R}$ software environment using numerical procedures such as Newton-Raphson method. It should be noted that when $p=2$ it (3) becomes half normal distribution.

\subsection{Order Statistics from Half Exponential Power}

Suppose that the $\left(X_{1}, \ldots, X_{n}\right)$ are jointly distributed random variables from (3). The corresponding order statistics are the $X^{\prime} s$ arranged in nondecreasing. The smallest of the $X_{i}$ is denoted by $X_{1: n}$, the second $X_{2: n}, \ldots$, and, finally the largest $X_{n: n}$. Thus we have 
$X_{1: n} \leq X_{2: n}, \ldots, \leq X_{n: n}$. Following the definition of cdf of order statistics $F_{i: n}(x)$, then we obtain for $F_{i: n}$ for a random variable having pdf (1) as

$$
\sum_{r=i}^{n}\left(\begin{array}{l}
n \\
r
\end{array}\right)[F(x)]^{r}[1-F(x)]^{n-r}=\sum_{r=i}^{n}\left(\begin{array}{l}
n \\
r
\end{array}\right)\left[\frac{\gamma\left(\frac{1}{p}, \frac{x^{p}}{p \sigma^{p}}\right)}{\Gamma\left(\frac{1}{p}\right)}\right]^{r}\left[1-\frac{\gamma\left(\frac{1}{p}, \frac{x^{p}}{p \sigma^{p}}\right)}{\Gamma\left(\frac{1}{p}\right)}\right]^{n-r}
$$

differentiating (6) with respect to $x$ and using the expression

$$
\int_{0}^{F(x)} \frac{n !}{(i-1) !(n-i) !} t^{i-1}(1-t)^{n-i} d t
$$

where $F(x)$ is as defined in (4), then the we obtain the corresponding pdf of the order statistics $f_{i: n}$ as

$$
\frac{n !}{(i-1) !(n-1) !}\left[\frac{\gamma\left(\frac{1}{p}, \frac{x^{p}}{p \sigma^{p}}\right)}{\Gamma\left(\frac{1}{p}\right)}\right]^{i-1}\left[1-\frac{\gamma\left(\frac{1}{p}, \frac{x^{p}}{p \sigma^{p}}\right)}{\Gamma\left(\frac{1}{p}\right)}\right]^{n-i} \frac{p^{1-\frac{1}{p}}}{\sigma \Gamma\left(\frac{1}{p}\right)} e^{-\frac{x^{p}}{p \sigma^{p}}}
$$

where $f_{i: n}(x)$ is the pdf of order statistics from (3). The $r t h$ moment $\mu_{i: n}^{r}$ can be obtained using the relation

$$
\mu_{i: n}^{r}=E\left(X_{i: n}^{r}\right)=\int_{-\infty}^{\infty} f\left(x_{i: n}\right) d x
$$

There was no close form solution to the relation, but $\mu_{i: n}^{(1)}$ corresponds to the first moment which is the mean of the order statistics and $\mu_{i: n}^{(2)}$ corresponds to the second moment, then the variance of the order statistics could be obtained from the defined relation

$$
\operatorname{var}\left(x_{i: n}\right)=\mu_{i: n}^{(2)}-\left[\mu_{i: n}^{(1)}\right]^{2}
$$

The aforementioned relations will be used to obtain the statistic from the sample data, which will be estimated numerically using $\mathrm{R}$ software tools.

\section{Data Analysis and Discussion of Results}

The data used was a secondary data from Ibadan Electricity Distribution Company (IBEDC), in Nigeria. The data were based on the first time failure of already installed new transformers. When the newly acquired transformer is installed and connected to various households and industries, the first time the transformer experience the first failure which required major repair are recorded, also the manufacturer of such transformer and other information are put into record. The concern of this research is to obtain model that will give a good fit to the first time failure of the transformers acquired. This with aims of predicting the first time failure of the newly acquired by the government. Though, there were many unnatural factors responsible for the breakdown such as: overloading more than manufacturer prescriptions, accident, pilferages, etc. in these cases we needed data cleansing in order to have reliable results. The analysis gave information on the life span of transformers between the year installed and the year of its first repaired.

The Figure 1 showed the line of fit for the model against the histogram plot of the first time failure of the transformers installed, It is usual practice in literature to assume half normal distribution for instance in the Figure 1, the dotted green line is the fitted 
line of a Half normal distribution (assumption) while the thick red line fit the Half Exponential distribution. Obviously, the half exponential power gave the best fit when compared to half normal distribution. This is due to shape $p$ in the half exponential power. The shape parameter $p$ made the distribution more flexible in the tail and made it more appealing analysis of data than the usual normal counterpart.

\section{Histogram of transformer lifetime}

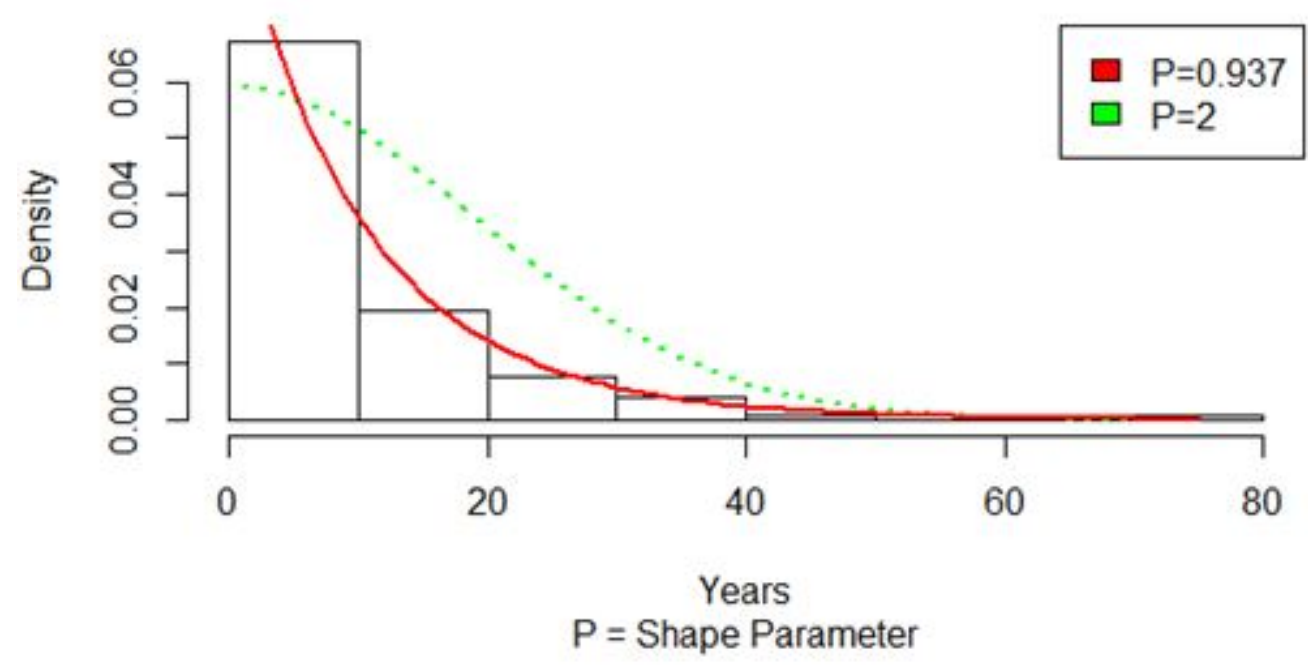

Figure 1: Data Plot on Transformer Lifetime

Parameters of the model were estimated through optimization method using $\mathrm{R}$ codes, the method gave the maximum likelihood estimation of the parameters for the cases of half normal and half exponential power distributions. But he best fit was obtained from the half exponential power distribution with scale and shape parameters 10.444275 and 0.937 respectively. For comparative purpose and for justification of half exponential power distribution, the Half normal distribution with shape parameter 2 was also fitted to the histogram.

\section{Conclusion}

In conclusion, the half exponential power distribution is more appealing and gives a better fit when compared with the half normal distribution in analysis of positively skewed first time failure of power distribution transformers due to the presence of shape parameter $p$, which gave it more flexibility than the normal distribution.

\section{References}

Agro, G. (1995). Maximum likelihood estimation for the exponential power function parameters, Communications in Statistics-Simulation and Computation 24(2): 523536. 
Gui, W. (2013). Statistical inferences and applications of the half exponential power distribution, Journal of Quality and Reliability Engineering 2013: 1-10.

Kalbfleisch, J. D. and Prentice, R. L. (2011). The statistical analysis of failure time data, Vol. 360, John Wiley \& Sons, New York (US).

Lawless, J. F. (2011). Statistical models and methods for lifetime data, Vol. 362, John Wiley \& Sons, New York (US).

Mineo, A. M. and Ruggieri, M. (2005). A software tool for the exponential power distribution: The normalp package, Journal of Statistical Software 12(4): 1-24.

Olosunde, A. (2013). On exponential power distribution and poultry feeds data: a case study, Journal of The Iranian Statistical Society 12(2): 253-270.

Olosunde, A. and Adegoke, A. (2016). Goodness-of-fit-test for exponential power distribution, American Journal of Applied Mathematics and Statistics 4(1): 1-8. 